\title{
Design of an holographic off-axis calibration light source for ARGOS at the LBT
}

\author{
Christian Schwab ${ }^{1, a}$, Wolfgang Gässler ${ }^{2}$, Diethard Peter ${ }^{2}$, Thomas Blümchen ${ }^{2}$, Simon Aigner ${ }^{3}$, and \\ Andreas Quirrenbach ${ }^{1}$ \\ 1 ZAH-Landessternwarte, Universität Heidelberg, Germany \\ 2 Max-Planck Institut für Astronomie, Heidelberg \\ 3 DIOPTIC GmbH, Bensheim, Germany
}

\begin{abstract}
We report on the design of an artificial light source for ARGOS, the multiple Rayleigh laser guide star (LGS) facility at the Large Binocular Telescope (LBT). Our light source mimics the expected night-time illumination of the adaptive secondary mirror (ASM) by the laser beacons very accurately and provides a way to check the achieved performance, allowing thorough testing of the system during day time. The optical design makes use of computer generated holograms (CGH) and strong aspheres to achieve a very small residual wavefront error. Additional structures on the CGH facilitate quick and precise alignment of the optics in the prime focus. We demonstrate that the scheme can be applied to the current European Extremely Large Telescope (E-ELT) design in a similar way.
\end{abstract}

\section{Introduction}

Future adaptive optics (AO) systems for ELTs face additional challenges compared to existing systems. The deformable mirrors (DMs) are directly incorporated into the telescope design and will therefore be in a fast beam, and the AO systems use more than one laser beacon in order to correct a large field of view [1-3]. LBT's ARGOS facility will incorporate all of these characteristics already.

A system as complex as a multi-wavefront sensor (WFS) AO module has many parameters, and uses a large number of interfaces. Thorough calibration of the components is necessary to understand their behavior and the interplay between them, and consequently tune the system to get optimal performance. It is highly desirable to have artificial light sources for calibrations during the day, allowing for extensive checking and adjusting of the system.

An ideal calibration light source provides the means to

- run the system in closed loop for tweaking and testing,

- evaluate the image quality in the focal plane in real time,

- measure the interaction matrix for night time operation,

- measure non-common path aberrations to the science camera.

We want to be able to distinguish between problems with the DM or the WFS and co-located optics by checking the image quality and measuring the achieved science light path wavefront. This also allows us to evaluate the performance of the system during testing (e.g. while running a closed loop). To accommodate for that, we provide a diffraction limited on-axis light source of stable and high wavefront quality, in the spectral region the science camera and the on-axis natural guide star WFS are designed for. This high order on-axis WFS can then be used as truth sensor.

An optical design that complies with the points above is challenging. In addition to very tight optical constraints, the unit has to be light, small and work in a wide temperature range. Speed and ease of operation are key to efficient and frequent use of the system. We thus aim for a very quick and automated deployment of the unit. To reliably measure the interaction matrix, and to assess the non common path errors, the light source needs to provide a wavefront as similar to that of the laser beacons

\footnotetext{
a e-mail: cschwab@lsw.uni-heidelberg.de
} the original work is properly cited. 


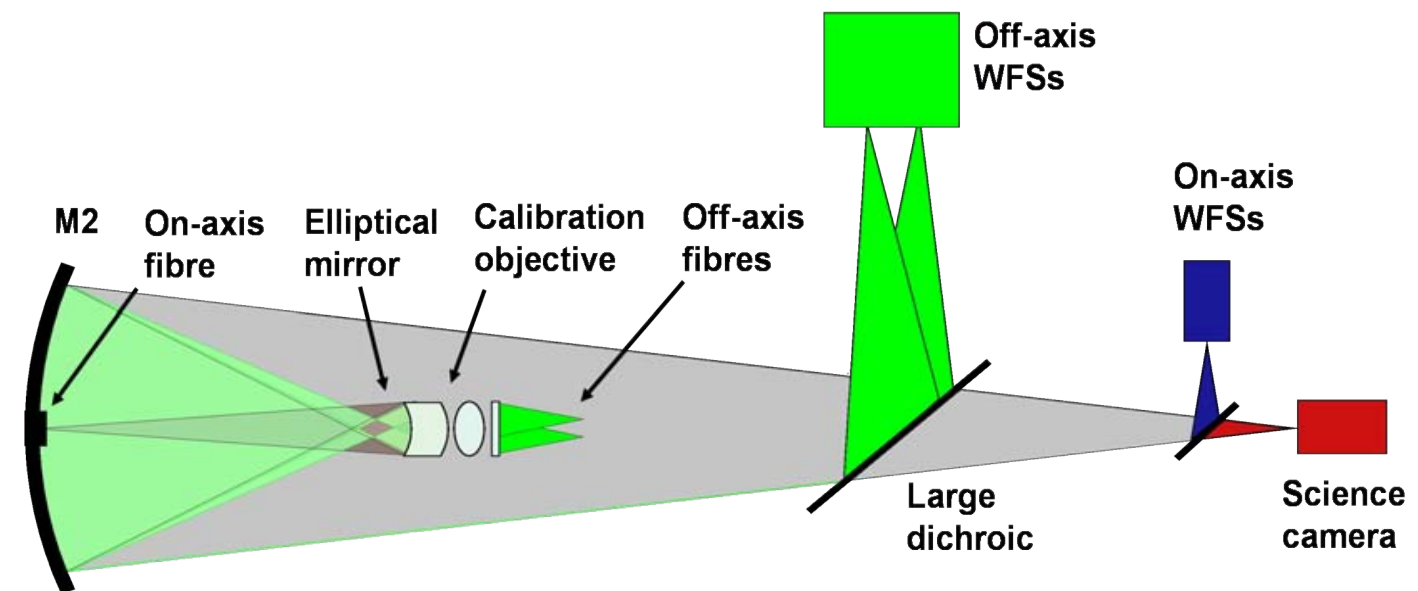

Fig. 1. Light paths of the calibration light sources.

as possible. The expected residual error the AO system achieves on sky constrains the difference in wavefront we can allow between the calibration source and the artificial laser guide stars; in our case we specified a maximal (static) deviation of $1 \lambda$, with an uncertainty of $\lambda / 10$ (at the LGS wavelength of $532 \mathrm{~nm})$.

Such a calibration light source is only possible if the beam has a waist before the deformable mirror (DM), where the calibration source can be inserted. While the Gregorian design of the LBT conveniently has a real prime focus, providing a plane conjugated to the WFSs before the adaptive secondary, a light source there has to copy the severe aberrations introduced by the f/1.14 parabolic primary. For a guide star at $12 \mathrm{~km}$ height and 2 arcmin off axis, as in the case of ARGOS, these are primarily several hundred waves of coma.

\section{Calibration scheme}

We project three fibres, one per beacon, through a compact f/1 objective onto the secondary (see Fig.1). The fibre images in the prime focus serve as input for the LGS WFSs. Additionally, a single on-axis fibre is mounted in the central hub of the adaptive secondary. It is imaged by reflection on the front surface of the lens onto the natural guide star (NGS) AO WFS and the science camera, where the focal plane point spread function (PSF) can be evaluated. This is important as the non-common path between the LGS WFSs and the NGS WFS or the science camera, respectively, contains a considerable number of optical elements, including a large ( $>20$ inches) dichroic and the fast pupil reimaging optics in front of the LGS WFSs.

\section{Mechanics}

The objective will be mounted below the adaptive secondary of LBT on a dedicated swing arm (Fig.2). Insertion of the arm into nominal position takes less than a minute. As the optics are light-weight, the arm has mainly to support itself. We choose carbon fibre reinforced plastic (CFRP) tube as construction material for its excellent stiffness and low weight, easing the construction of the insertion mechanism. The four meter long arm is automated by an electro motor coupled to a harmonic drive gear. Differential thermal expansion between the swing arm and the ASM's aluminium arm, resulting in a mismatch in radial position of a few millimetres under extreme temperature conditions, can be counterbalanced by the ASM hexapod. Finite elements analysis (FEA) shows that the flexure of the structure does not exceed $1 \mathrm{~mm}$ for zenith angles of up to $60^{\circ}$. Great care has been taken to reduce the vignetting of subapertures from the front part of the arm that intrudes into the beam. A structure of thin carbon plates minimizes the footprint on individual subapertures. 


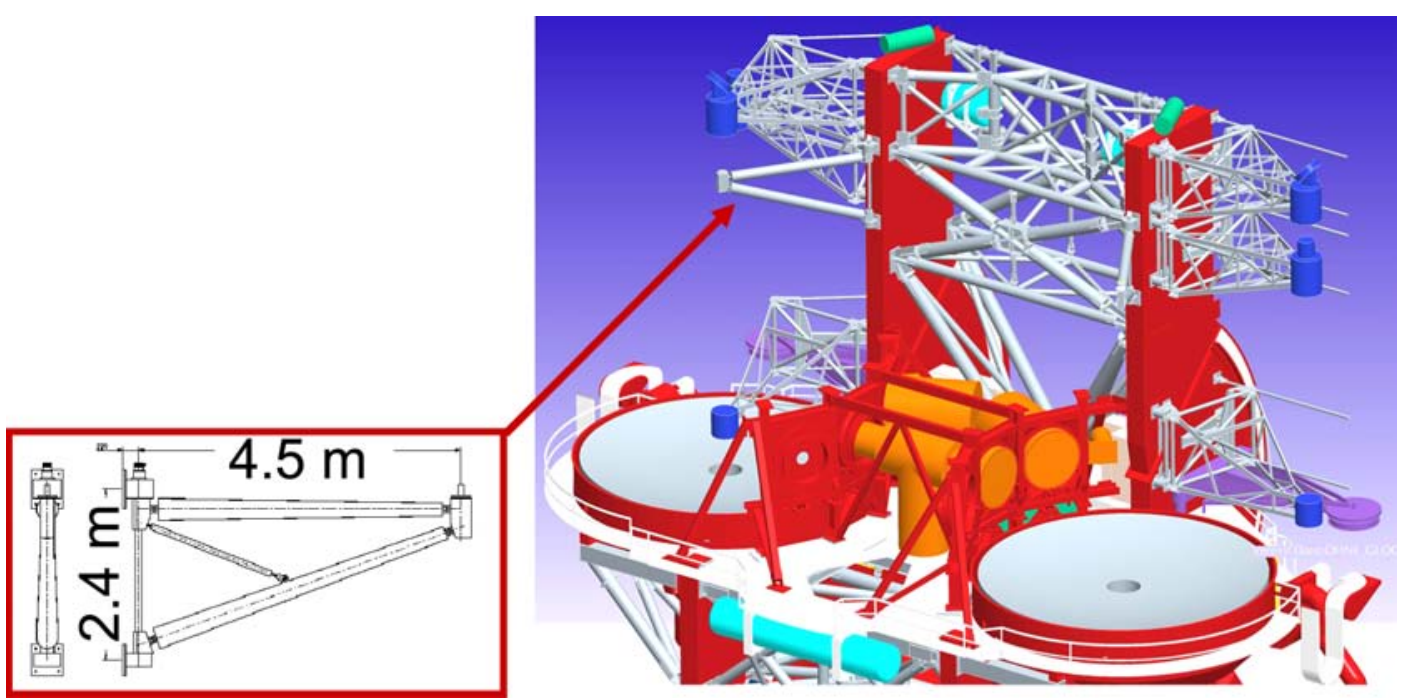

Fig. 2. The CFRP swingarm's dimensions and location at the LBT.

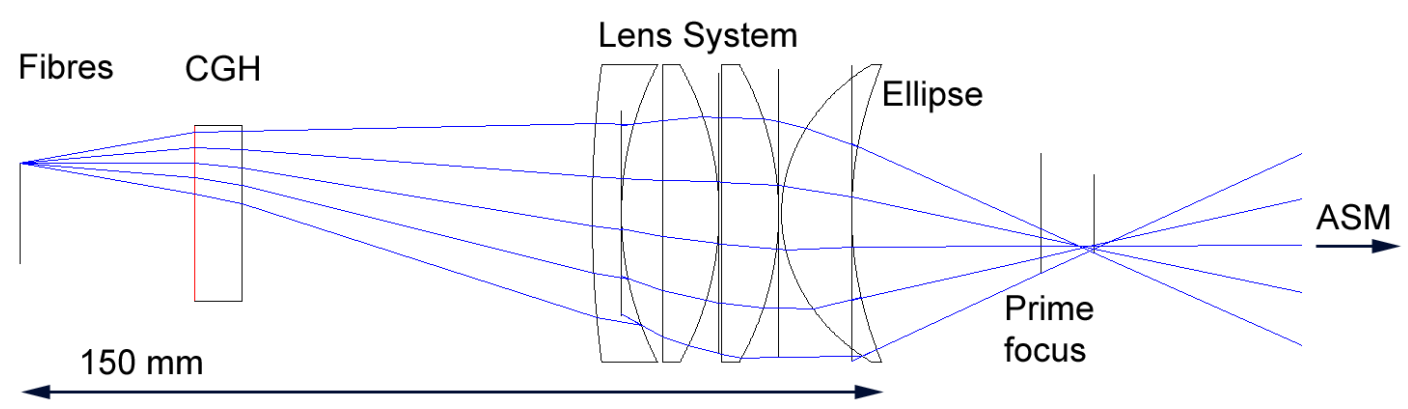

Fig. 3. Optical layout of the calibration objective.

\section{Optical Design}

The optical design provides three off-axis and one on-axis source simultaneously. To generate the offaxis sources the calibration unit's objective uses four 2 inch lenses (Fig.3). Three of them are standard catalogue lenses made from BK7 glass, while the fourth is derived from a standard lens by making one surface aspheric. The lenses in front of the CGH have two functions: to achieve the desired fast focal ratio of the beam, and to provide a plane for the hologram where the three beams are separated, but the rays within each beam are not scrambled, and fairly evenly distributed. The orientation of the design on catalog lenses allows us to build an inexpensive prototype by substituting the asphere with a simple sphere, to evaluate the performance of our design and check for possible assembly problems.

We use a custom CGH as we found no conventional optical solution to achieve the desired wavefront. Together with the lens system, the CGH adjusts the wavefront of the off-axis fibres to have the same aberrations as those produced by the primary, with a residual of less than $\lambda / 10$ (Fig.4). A CGH is the ideal tool to produce the non-axisymmetric wavefront we need. As we use a laser for our artificial guide star, the need to operate a $\mathrm{CGH}$ in monochromatic light is easy to satisfy. Contrary to conventional optical elements, the missing axial symmetry presents no additional challenge for the design of the fabrication. Even large aspheric deviations can be produced with high precision, and the use of multiple diffractive alignment structures written on the same substrate permits the critical alignment with respect to the other optical elements (see for example [4] and references therein). Our design uses three CGH structures, one per WFS, written on the same substrate. Additional alignment structures 

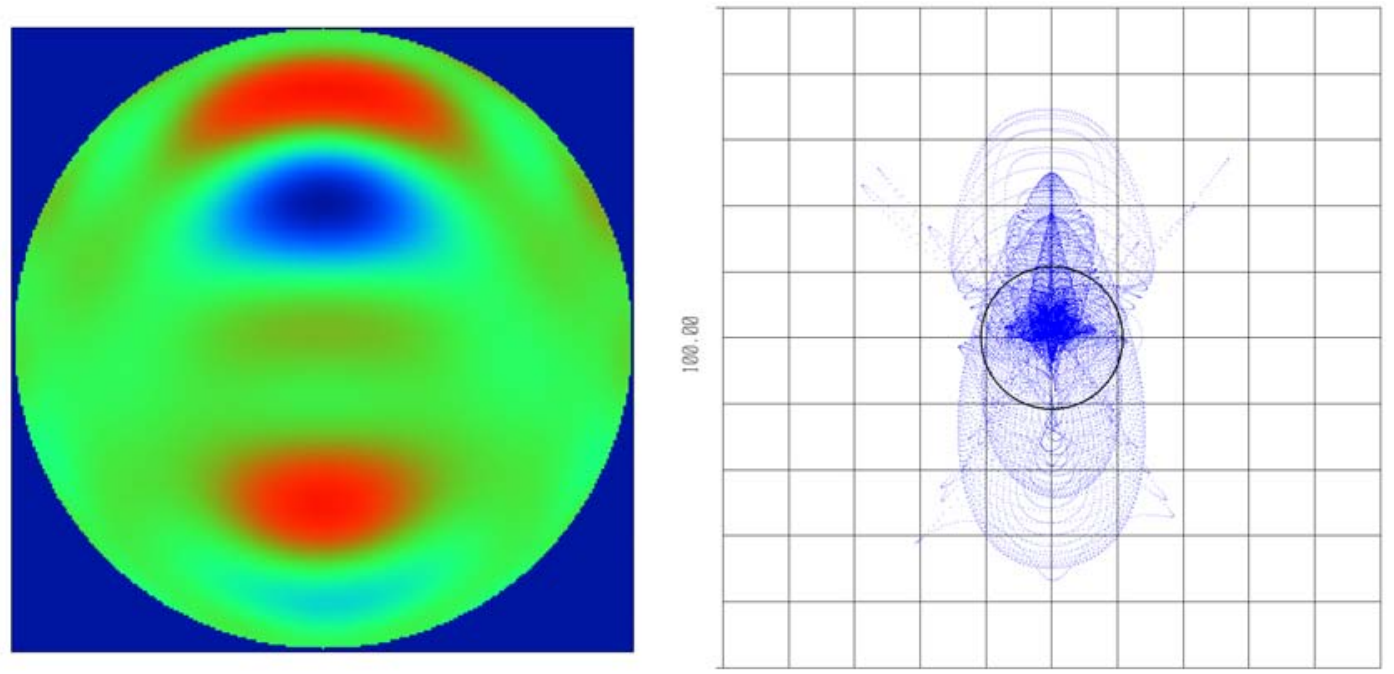

Fig. 4. Left: Wavefront as created by the off-axis calibration light source. The wavefront error is $0.48 \lambda \mathrm{PV}$ and $0.07 \lambda$ RMS. Right: Spot diagram on the LGS WFS. RMS spot radius is $12.4 \mu \mathrm{m}$. For comparison, the Airy disc radius shown is $11.3 \mu \mathrm{m}$

are used to align the fibres to the $\mathrm{CGH}$, the $\mathrm{CGH}$ to the lenses, and for an initial positioning of the calibration objective with respect to the secondary mirror.

The on-axis source is designed to make use of only one optical element, to keep the wavefront insensitive to misalignments and temperature changes. The lens surface closest to the secondary is a f/0.5 ellipse. One focus coincides with the prime focus, and the other with the tip of the on-axis fibre in M2. This fibre is coupled to a tungsten lamp. A dichroic coating on the ellipse reflects wavelengths longer than the $532 \mathrm{~nm}$ used for the off-axis lightsource, forming an image of the fibre in the prime focus of the telescope. The image is achromatic and diffraction limited. In fact, the 2 inch diameter ellipse is considerably oversized, so the aperture stop of the system is the secondary mirror, just like at night. The $9 \mu \mathrm{m}$ diameter fibre (Corning SMF 28e) is demagnified by a factor of 25; the geometrical image is much smaller than the airy disc diameter. We achieve excellent thermal stability by using Clearceram as material for the lens with the ellipsoidal surface. Coputer numerical controlled (CNC) grinding and polishing, followed by subsequent magnetorheological finishing (MRF) yields a surface accuracy of approximately $\lambda / 10$ peak to valley [5].

Aligning the calibration objective in the prime focus to the necessary accuracy of some $10 \mu \mathrm{m}$ is challenging. We will use the on-axis spot as alignment aid for the whole unit. After inserting the swing arm, placing the calibration unit roughly into the right position, the on-axis source is switched on and the wavefront is measured with the natural guide star wavefront sensor. The secondary mirror is then iteratively repositioned with its hexapod until the wavefront error is less than $\lambda / 10$. To double-check the position, a further alignment structure on the CGH provides an additional focus that, in the case of correct alignment, is coincident with the on-axis lightsource focus. No external position measurement is necessary.

\section{Considerations for the E-ELT}

We investigate the transferability of our calibration scheme to the E-ELT based on the amount of aberrations of the off-axis LGS images in the intermediate focus. This approach is valid as the amount of abberations translates to the phase variation the $\mathrm{CGH}$ has to provide; the CGH's capabilities are to zeroeth order not dependent on the type of abberation. The optical design for the E-ELT has an intermediate cassegrain focus [1]. This position is ideal for a calibration light source as it is easily accessible. Fig.5 shows the spot diagram in that focus for an object at a height of $92 \mathrm{~km}, 2$ arcmin 

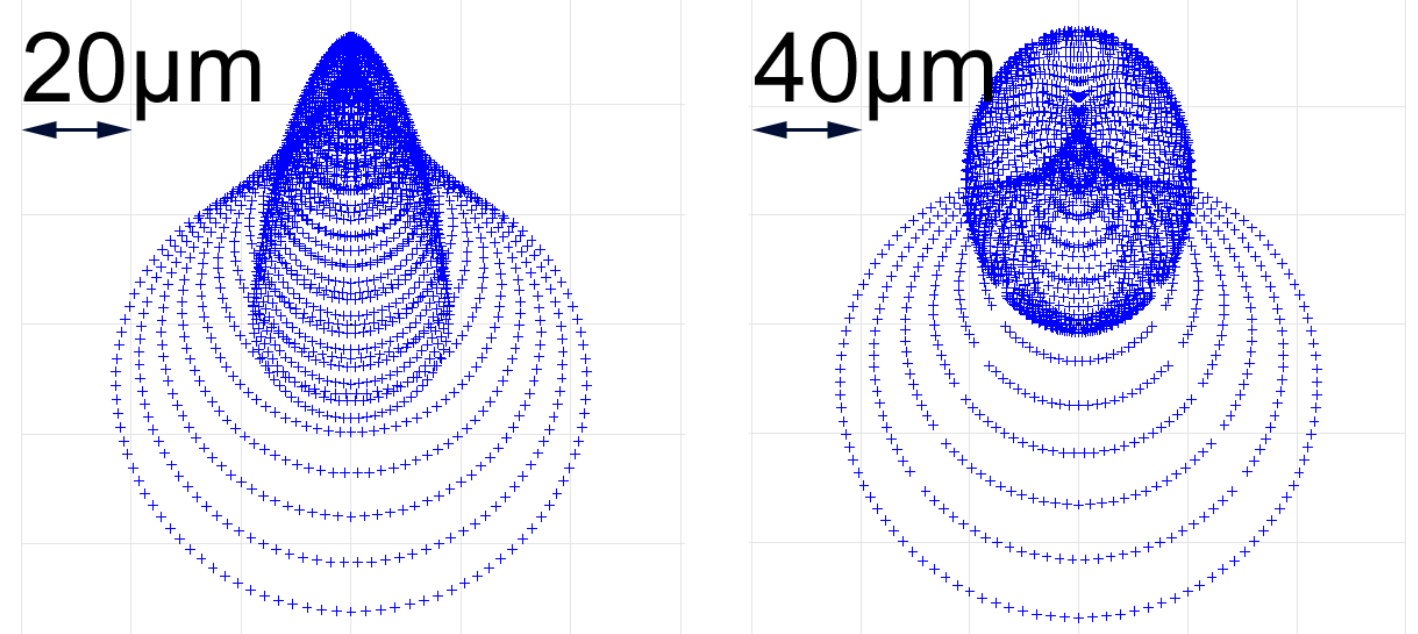

Fig. 5. Left: Spot diagram of a rayleigh laser beacon in LBT's focal plane. Right: Spot diagram of a sodium laser beacon in the intermediate focus of the E-ELT. Both sources are 2 arcmin off-axis. Note the difference of a factor 2 in scale.

off-axis, in comparison to the spot of the Rayleigh LGS in LBT's prime focus. While the dominating aberrations differ, the total wavefront deformation and the spot size are comparable. As the E-ELT's intermediate focus lies within M4, the space is more easily accessible. We think that a CGH-corrected objective would be the ideal solution to feed the AO systems with a well-corrected calibration beam. We note that the GMT's optical layout permits the use of such a solution in the same position as at the LBT, too [2].

\section{Conclusions}

We presented our concept for the AO calibration system at LBT. It employs custom CGHs to imitate the illumination of the DM by the laser guide stars. This makes it possible to calibrate and improve the system's performance during daylight hours. In particular, it allows us to measure the interaction matrix between WFS and DM. We foresee a similar system being an indispensable part of AO facilities on future large telescopes. As outlined above, our optical concept is directly transferrable to the E-ELT.

\section{References}

1. Cuby, J.-G., Morris, S., Bryson, I., et al., in Ground-based and Airborne Instrumentation for Astronomy II. (Proceedings of the SPIE, 2008) pp. 70141K-70141K-11

2. Johns, M., Angel, J. R. P., Shectman, S., et al., in Ground-based Telescopes. (Proceedings of the SPIE, 2004) Volume 5489, pp. 441-453

3. Boyer, C., Ellerbroek, B., Gedig, M., et al., in Adaptive Optics Systems. (Proceedings of the SPIE, 2008) pp. $70152 \mathrm{~N}-70152 \mathrm{~N}-12$

4. Zehnder, R. and Burge, J. H., Zhao, C., in Optomechanical Technologies for Astronomy. (Proceedings of the SPIE, 2006) Volume 6273, pp. 62732S

5. Tricard, M., Dumas, P., Forbes, G., in Optical Design and Testing II. (Proceedings of the SPIE, 2005) Volume 5638, pp. 284-299 